\title{
Expressions for Expectations and Variances of Cycle Times for Yard Cranes by Considering Dependencies among Time Elements
}

\author{
Byung Kwon Lee \\ Department of Industrial Engineering, Pusan National University, Busan 609-735, Korea \\ Tel: +82-51-510-1483, Fax: +82-51-512-7603, E-mail: errorplus@pusan.ac.kr \\ Kap Hwan Kim ${ }^{\dagger}$ \\ Department of Industrial Engineering, Pusan National University, Busan 609-735, Korea \\ Tel: +82-51-510-2419, Fax: +82-51-512-7603, E-mail: kapkim@pusan.ac.kr \\ Won Young Yun \\ Department of Industrial Engineering, Pusan National University, Busan 609-735, Korea \\ Tel: +82-51-510-2421, Fax: +82-51-512-7603, E-mail: wonyun@pusan.ac.kr
}

Received, March 26, 2011; Revised, September 14, 2011; Accepted, September 21, 2011

\begin{abstract}
During the design process of a terminal, the handling capacity of a container yard needs to be evaluated in advance. This study suggests formulas for estimating the expectations and the variances of cycle times for various types of operations of a yard crane. Statistical analysis is used to estimate the expectations and variances. The main focus of this study is to show the impact of interdependencies among handling time elements on the expectation and variance of the cycle times; these interdependencies have not been considered in previous studies. Numerical experiments are done for evaluating the difference in the variance of cycle times and the waiting of trucks between the cases with and without the consideration of interdependencies.
\end{abstract}

Keywords: Container Terminal, Cycle Time, Yard Crane, Statistical Analysis

\section{INTRODUCTION}

Container terminals play an important role in international transportation by serving as multi-modal interfaces between sea and land transport. Three different types of handling equipment are usually used at container terminals: quay cranes (QCs), yard cranes (YCs), and internal trucks. The handling operations at container terminals are three types: vessel operations associated with containerships, receiving/delivery operations of trucks, and container handling and storage operations in the yard. Vessel operations include unloading and loading operations. In unloading operations, containers are discharged from the vessel and stacked in a marshalling yard. In loading operations, containers are transported in the opposite direction. During the unloading operation, QCs transfer containers from a ship to internal trucks. The internal trucks then deliver the inbound (import/ unloading) containers to YCs, which picks them up and stacks them in specific positions in a marshalling yard.
During the loading operation, this unloading process is carried out in the opposite direction.

For efficiency, sets of adjacent vessel slots are usually allocated to outbound containers of the same group -containers of the same size and bound for the same destination port-in the stowage plan of a vessel. Thus, a QC tends to load containers of the same group consecutively. Therefore, for efficient yard operation, the storage plans for outbound containers are prepared in advance and empty stacks adjacent to each other or in a whole yard bay are pre-assigned to outbound containers of the same group in many container terminals (Chen, 1999). Using this grouped layout of outbound containers, a YC can typically pick up 10 30 outbound containers of the same group consecutively from the same yard bay, which are delivered to a QC to be loaded onto a collection of adjacent vessel slots. During the unloading operation, a YC usually stacks inbound containers into an empty yard bay until the yard bay becomes full, after which they are moved to the next empty yard bay.

$\uparrow$ : Corresponding Author 
During the receiving operation, when a container arrives at a terminal via a truck, the location where the outbound container is to be stored is assigned to the truck. When the truck arrives at the transfer point (TP), a YC receives the container from the truck. During the delivery operation, the arriving truck is directed to the block with the inbound container requested by the truck.

Bozer and White (1984) have proposed formulas for estimating the travel times during single- and dualcycle operations of a storage and retrieval $(\mathrm{S} / \mathrm{R})$ machine whose movement is characterized by Tchebychev travel. Bozer and White (1990) have suggested a method for estimating the throughput rate of an S/R machine in endof-aisle order-picking systems. Hwang and Lee (1990) have developed travel-time models that consider more realistic operating characteristics of the $S / R$ machine, including acceleration and deceleration. Le-Duc (2005) has proposed a travel-time model for the $\mathrm{S} / \mathrm{R}$ machine and conveyor simultaneously.

Hu et al. (2005) have proposed an expected traveltime model for a type of automatic storage and retrieval system (AS/RS) that can be used for container storage at container terminals. By using a Markov-chain model and a mathematical approximation, Vidovic and Kim (2006) have proposed a method for estimating the cycle time of three-stage, material handling systems consisting of a QC, YC, and multiple trucks. Kim (1997) has proposed a formula for estimating the total number of handling occurrences, including re-handling, required to retrieve all the inbound containers that are stacked in a yard bay. de Castilho and Daganzo (1993) have proposed simple mathematical equations for the expectations and variances of the number of relocations required to retrieve a container from a yard bay. Kim (2006) has estimated the expected YC cycle times for storage/retrieval operations. However, he has simplified the operations of container terminals by assuming random storage and retrieval and has not considered the characteristics of different types of operations such as loading, unloading, receiving, and delivery. Lee and Kim (2010a) have derived the expressions for the expectation and the variance of the cycle times of various types of operations of yard cranes. Lee and Kim (2010b) have proposed analytic models for determining the optimal configuration: blocks for which TPs are located beside each bay and blocks for which TPs are located at both ends. Under the assumption that the trolley and gantry movements can be undertaken simultaneously, they have derived formulas for the expected values and variances of the cycle times for different types of operation.

The purpose of this paper is to analyze the effect of interdependencies among the handling elements constituting an operation cycle of a $\mathrm{YC}$ on various performance measures of YCs. The contributions of this paper are as follows.

(1) Expressions for the covariance of interdependent handling elements of an operation cycle are proposed for two different traveling modes of trolleys (rectangular and Tchebychev movements of YCs) and two types of layouts (blocks laid parallel to the quay and blocks laid perpendicular to the quay).

(2) Statistical analysis and characterization of the effects of interdependencies among the handling elements for four types of operations (receiving, loading, unloading, and delivery), two block layouts (parallel and perpendicular block layouts), and two traveling modes of trolley (rectangular and Tchebychev movements).

(3) Evaluation of the impact of interdependencies among the handling elements on values of the expectation and the variance of cycle times by conducting numerical experiments.

The remainder of this paper is organized as follows. Section 2 introduces expressions for the expected cycle times and variances for different types of operations. Section 3 analyzes the interdependencies between time elements, which constitute an operation cycle of a YC, and compares values of the variances of cycle times between the case without and with the consideration of the covariance among the time elements. Numerical experiments are described in section 4. Conclusions are reported in section 5 .

\section{DEVELOPING CYCLE TIME MODELS FOR YARD CRANE}

The cycle time depends on the type of $\mathrm{YC}$ and its technical specifications. YC movements are classified into three types: gantry travel, trolley movement, and hoisting (or lowering) of the spreader. For safety reasons, it may be assumed that the movements are carried out sequentially. In other words, the trolley movement begins after the hoisting movement is completed. After the trolley movement is completed, the gantry travel begins. This type of movement is referred to as "rectangular movement." However, to speed up the movement, the trolley movement and the gantry travel may be carried out simultaneously, which is called "Tchebychev movement." Even in this case, the hoisting movement may not be combined with other movements. Formulas for the expectations and variances of the cycle time during the receiving, loading, unloading, and delivery operations are derived below. All the contents in this section are reproduced from Lee and Kim (2010a).

\subsection{Basic Elements of Handling}

The following notational conventions are used.

\section{Constant or variable movement time}

$T_{z w}^{x y}=$ the handling time for the movement of a YC whose starting position, ending position, type of motion, and state of equipment (loaded or empty) are denoted as $x, y, z$, and $w$, respectively. It is a ran- 
dom variable.

$R_{W H}=$ the number of re-handles that are required to pick up a random container from a bay with $W$ rows and $H$ tiers. This is a random variable.

$t_{z w}^{x y}=$ the handling time for the movement of a YC obtained by replacing " $T$ " in $T_{z w}^{x y}$ with ' $t$ ", when the travel distance is a constant rather than a random variable.

Type of motion (subscripts)

$g=$ Gantry travel

$t=$ Trolley travel

$h=$ Hoisting up or down

$r$ Releasing a container

$p=$ Picking up a container

$R=$ Re-handling operation

State of equipment (subscripts)

$e$ = Empty

$l=$ Loaded

\section{Starting and ending positions (superscripts)}

$a=$ the arbitrary (random) position within a range where a piece of handling equipment moves for picking up or releasing a container from or into the storage area.

$b=$ the boundary of a container stacking block.

$c=$ the position of the spreader that is picking up or releasing a container from or onto a chassis.

Other parameters

$W=$ the number of stacks in a bay.

$H=$ the number of tiers of stacks.

The following are some examples of the notations used for the handling elements. $T_{g e}^{a a}$ is the time required for an empty YC to execute gantry travel between two random positions. $T_{t l}^{c a}$ is the time required for a loaded trolley to move from the parking position of a chassis to a random position in a bay. The notation $t_{h e}^{b c}$ represents the time required for lowering an empty spreader from its top position to a container on a truck chassis, and $t_{p}$ represents the time that is required for a spreader to pick up a container.

We assume that $t_{t^{*}}^{b c} \leq t_{g^{*}}^{b b}$. Further, let $s_{1}=t_{t^{*}}^{b c} \leq t_{g^{*}}^{b b}$ and $s_{2}=t_{t^{*}}^{b c} \leq t_{g^{*}}^{b b}$; both $s_{1}$ and $s_{2}$ are less than unity. Some movements have constant time values, whereas others have stochastic time values because of the randomness in their associated positions. The stochastic time values of random movements can be classified into two types: $T_{* *}^{c a}$ and $T_{* *}^{a a}$. In Tchebychev movements, two elementary movements are performed simultaneously. In the motion of YCs, the simultaneous movement of the gantry and trolley are included in this category. The time for the Tchebychev movement is the maximum of the trolleymovement time and the gantry-movement time, which is denoted by $\operatorname{Max}\left(T_{t^{*}}^{* *}, T_{g^{*}}^{* *}\right)$.

In summary, there are four handling elements whose time values are stochastic: $T_{* *}^{c a}, T_{* *}^{a a}, \operatorname{Max}\left(T_{t^{*}}^{c a}, T_{g^{*}}^{a a}\right)$, and. Note that $T_{* *}^{c a}$ and $T_{* *}^{a c}$ have the same statistical characteristics and, thus, the same mean and variance. The expected values and variances of these four random variables can be derived as follows.

$$
\begin{aligned}
& E\left(T_{* *}^{c a}\right)=\frac{t_{*}^{c b}}{2} . \\
& \operatorname{Var}\left(T_{* *}^{c a}\right)=\frac{\left(t_{* b}^{c b}\right)^{2}}{12} . \\
& E\left(T_{* *}^{a a}\right)=\frac{t_{*}^{b b}}{2} . \\
& \operatorname{Var}\left(T_{* *}^{a a}\right)=\frac{\left(t_{* *}^{b b}\right)^{2}}{18} . \\
& E\left[\operatorname{Max}\left(T_{t^{*}}^{c a}, T_{g^{*}}^{a a}\right)\right]=t_{g^{*}}^{b b}\left(-\frac{1}{12} s_{1}^{3}+\frac{1}{3} s_{1}^{2}+\frac{1}{3}\right) . \\
& \operatorname{Var}\left[\operatorname{Max}\left(T_{t^{*}}^{c a}, T_{g^{*}}^{a a}\right)\right]=\left(t_{g^{*}}^{b b}\right)^{2} \\
& \qquad\left[-\frac{1}{10} s_{1}^{4}+\frac{1}{3} s_{1}^{3}+\frac{1}{6}-\left(-\frac{1}{12} s_{1}^{3}+\frac{1}{3} s_{1}^{2}+\frac{1}{3}\right)^{2}\right] . \\
& \operatorname{E}\left[\operatorname{Max}\left(T_{t^{*}}^{a a}, T_{g^{*}}^{c a}\right)\right]=t_{g^{*}}^{c b}\left(\frac{1}{12} s_{2}^{2}+\frac{1}{2}\right) . \\
& \operatorname{Var}\left[\operatorname{Max}\left(T_{t^{*}}^{a a}, T_{g^{*}}^{c a}\right)\right]=\left(t_{g^{*}}^{c b}\right)^{2}\left[\frac{1}{15} s_{2}^{3}-\left(\frac{1}{12}\right)^{2}\left(s_{2}^{4}+12 s_{2}^{2}-12\right)\right] .
\end{aligned}
$$

The detailed derivation of the above expressions has been provided by Lee and Kim (2010a). To improve the accuracy of estimation, modified versions of the previous general formulas are used in the calculations for the following three cases:

$$
E\left(T_{t^{*}}^{c a}\right)=t_{t^{*}}^{c b}-t_{t^{*}}^{b b}+\frac{t_{t^{*}}^{b b}}{2}=t_{t^{*}}^{c b}-\frac{t_{t^{*}}^{b b}}{2},
$$

which is slightly larger than $t_{t^{*}}^{c b} / 2$;

$$
E\left(T_{h^{*}}^{c a}\right)=t_{h^{*}}^{b b}-t_{h^{*}}\left(\frac{H+1}{2}\right) ;
$$

and

$$
\operatorname{Var}\left(T_{h^{*}}^{c a}\right)=\left(t_{h^{*}}\right)^{2}\left(\frac{H^{2}-1}{2}\right)
$$

Where $t_{h^{*}}$ is the time for the vertical movement (hoisting/lowering) in relation to the height of a container. Note that, in expressions (9), (10), and (11), we assume that containers are stacked at their full tier heights. However, when the height of container stacks is less than their full tier heights, the values of $t_{t^{*}}^{c b}, t_{t^{*}}^{b b}$, and $H$ can be adjusted accordingly by considering the maximum height of the stacks as the full tier height in the expression for hoisting/lowering of the spreader.

During the delivery operation, because trucks often randomly request for inbound containers, re-handling operations become necessary. Thus, for estimating the cycle time of delivery operation, it is necessary to estimate the number of re-handles for a delivery order. Under the assumption that container retrievals are performed in a random order, Kim (1997) has derived a formula for estimating the expected number of re-handles $\left(E\left(R_{W H}\right)\right)$ per pickup (when all containers are retrieved 
from a bay). He has expressed it as a simple equation in terms of the number of rows and tiers. The variance of the number of re-handles $\left(\operatorname{Var}\left(R_{W H}\right)\right)$ for a random container retrieval has been derived by Lee and Kim (2010a).

\subsection{Rectangular Movement in Parallel Block Layout}

This section assumes that YCs move in a rectangular manner, which means that the gantry and the trolley movements are not performed simultaneously. The time elements of handling can be listed sequentially. The set of time elements consisting of a receiving operation, $S_{R}$, is $\left\{T_{g e}^{a a}, T_{t e}^{a c}, t_{h e}^{b c}, t_{p}, t_{h l}^{c b}, T_{t l}^{c a}, T_{h l}^{b a}, t_{r}, T_{h e}^{a b}\right\}$. The set of time elements consisting of a loading operation, $S_{L}$, is $\left\{T_{g e}^{a a} N\right.$ $\left.\left(1 / l_{0}\right), T_{t e}^{c a}, t_{h e}^{b a}, t_{p}, t_{h l}^{a b}, T_{t l}^{a c}, t_{h l}^{b c}, t_{r}, T_{h e}^{c b}\right\}$. The set of time elements consisting of an unloading operation, $S_{U}$, is $\left\{T_{g e}^{a a}\right.$ $\left.N\left(1 / u_{W H}\right), T_{t e}^{a c}, t_{h e}^{b c}, t_{p}, t_{h l}^{c b}, T_{t l}^{c a}, t_{h l}^{b a}, t_{r}, T_{h e}^{a b}\right\}$. The set of time elements consisting of a delivery operation, $S_{D}$ is $\left\{T_{g e}^{a a}\right.$, $\left.T_{t e}^{c a}, \sum_{j=1}^{R_{W H}} T_{U}^{j}, T_{h e}^{b a}, t_{p}, T_{h l}^{a b}, T_{t l}^{a c}, t_{h l}^{b c}, t_{r}, t_{h e}^{c b}\right\}$. The set of time elements consisting of a re-handling operation, $S_{R E}$ is $\left\{T_{h e}^{b a}, t_{p}, T_{h l}^{a b}, T_{t l}^{a a}, T_{h l}^{b a}, t_{r}, T_{h e}^{a b}, T_{t e}^{a a}\right\}$.

Note that represents a random variable with a value of 0 or 1 . The probability that $N(p)$ becomes 1 is $p$. Thus, $E[N(p)]=p$ and $\operatorname{Var}[N(p)]=p(1-p)$. For example, the handling element of $T_{g e}^{a a}$ can be observed once during $l_{0}$ loading operations, whereas the re-handling operation $T_{U}^{j}$ (the time for the $j^{\text {th }}$ rehandle) occurs $R_{W H}$ times during a delivery operation. Although $l_{0}$ can also be regarded as a random variable, we treat it as a constant for the sake of simplifying the analysis. Thus,

$$
E\left[T_{g e}^{a a} N\left(1 / l_{0}\right)\right]=\left(1 / l_{0}\right) E\left(T_{g e}^{a a}\right)
$$

Note

$$
\begin{gathered}
E\left(N\left(1 / l_{0}\right)\right)=\frac{1}{l_{0}} . \\
\operatorname{Var}\left(N\left(1 / l_{0}\right)\right)=\left(0-\frac{1}{l_{0}}\right)^{2}\left(\frac{l_{0}-1}{l_{0}}\right)+\left(1-\frac{1}{l_{0}}\right)^{2}\left(\frac{1}{l_{0}}\right)=\frac{l_{0}-1}{l_{0}^{2}} .
\end{gathered}
$$

Thus,

$$
\operatorname{Var}\left(T_{g e}^{a a} N\left(1 / l_{0}\right)\right)=\frac{1}{l_{0}} \operatorname{Var}\left(T_{g e}^{a a}\right)+E^{2}\left(T_{g e}^{a a}\right) \frac{l_{0}-1}{l_{0}^{2}} .
$$

With regard to the unloading operation, because containers are discharged consecutively into the same bay until no more stacking is possible, it is assumed that the operation is performed consecutively $u_{W H}$ times at the same bay. Note that $u_{W H}$ is the maximum number of containers that can be discharged into a yard bay and can be expressed as $u_{W H}=W H-(H-1)$. The term $(H-1)$ is deducted from $W H$ to account for the empty spaces that are necessary for relocations. Thus, gantry travel occurs at every ${u_{W H}{ }^{\text {th }}}$ unloading operation. For the case of $T_{g e}^{a a} N\left(1 / u_{W H}\right)$, a similar calculation may be applied to derive the expectation and variance.

The expected value and variance of $T_{R}$ may be derived as follows:

$$
E\left(T_{R}\right)=E\left(R_{W H}\right) E\left(T_{U}\right)
$$

where $T_{U}$ is the cycle time of a re-handling operation. The variance of the cycle time for a re-handling operation is

$$
\operatorname{Var}\left(\sum_{j=1}^{R_{W H}} T_{U}^{j}\right)=E\left(R_{W H}\right) \operatorname{Var}\left(T_{U}\right)+E^{2}\left(T_{U}\right) \operatorname{Var}\left(R_{W H}\right) .
$$

\subsection{Rectangular Movement in Perpendicular Block Layout}

The time elements of handling can be listed as per the operational sequence during a cycle. $S_{R}=\left\{T_{g e}^{a c}, T_{t e}^{a a}\right.$, $\left.t_{h e}^{b c}, t_{p}, t_{h l}^{c b}, T_{g l}^{a a}, T_{h l}^{b a}, t_{r}, T_{h e}^{a b}\right\}, S_{L}=\left\{T_{g e}^{c a}, T_{t e}^{a a}, T_{h e}^{b a}, t_{p}, T_{h l}^{a b}, T_{g l}^{a c}\right.$, $\left.T_{t l}^{a a}, t_{h l}^{b c}, t_{r}, t_{h e}^{c b}\right\}, S_{U}=\left\{T_{g e}^{a c}, T_{t e}^{a a}, t_{h e}^{b c}, t_{p}, t_{h l}^{c b}, T_{g l}^{c a}, T_{t l}^{c a}, T_{h l}^{b a}, t_{r}, T_{h e}^{a b}\right\}$, $S_{D}=\left\{T_{g e}^{c a}, T_{t e}^{a a}, \sum_{j=1}^{R_{W H}} T_{U}^{j}, T_{h e}^{b a}, t_{p}, T_{h l}^{a b}, T_{g l}^{a c}, T_{t l}^{a a}, t_{h l}^{b c}, t_{r}, t_{h e}^{c b}\right\}, S_{R E}$ $=\left\{T_{h e}^{b a}, t_{p}, T_{h l}^{a b}, T_{t l}^{a a}, T_{h l}^{b a}, t_{r}, T_{h e}^{a b}, T_{t e}^{a a}\right\} . S_{R E}$ is the same as the set defined in section 2.2.

\subsection{Tchebychev Movement in Parallel Block Layout}

We have derived the expressions for the case of blocks with a parallel layout. The YCs travel under the Tchebychev metric, which means that the trolley of a YC moves while the YC travels in gantry direction. The time elements of handling can be listed as follows: $S_{R E}$ $=\left\{\operatorname{Max}\left(T_{t e}^{a c}, T_{g e}^{a a}\right), t_{h e}^{b c}, t_{p}, t_{h l}^{c b}, T_{t l}^{c a}, T_{h l}^{b a}, t_{r}, T_{h e}^{a b}\right\} . S_{L}=\left\{\operatorname{Max}\left(T_{t e}^{c a}\right.\right.$, $\left.\left.T_{g e}^{a a}\right) N\left(1 / l_{0}\right), T_{t e}^{c a}\left(1-N\left(1 / l_{0}\right)\right), T_{h e}^{b a}, t_{p}, T_{h l}^{a b}, T_{t l}^{a c}, t_{h l}^{b c}, t_{r}, t_{h e}^{c b}\right\}, S_{U}=$ $\left\{\operatorname{Max}\left(T_{t e}^{a c}, T_{g e}^{a a}\right) N\left(1 / u_{W H}\right), T_{t e}^{a c}\left(1-N\left(1 / u_{W H}\right)\right), t_{h e}^{b c}, t_{p}, t_{h l}^{c b}, T_{t l}^{c a}\right.$, $\left.T_{h l}^{b a}, t_{r}, T_{h e}^{a c}\right\}, S_{D}=\left\{\operatorname{Max}\left(T_{t e}^{c a}, T_{g e}^{a a}\right), \sum_{j=1}^{R_{W H}} T_{U}^{j}, T_{h e}^{b a}, t_{p}, T_{h l}^{a b}, T_{t l}^{a c}\right.$, $\left.t_{h l}^{b c}, t_{r}, t_{h e}^{c b}\right\}$, and $S_{R E}=\left\{T_{h e}^{b a}, t_{p}, T_{h l}^{a b}, T_{t l}^{d a}, T_{h l}^{b a}, t_{r}, T_{h e}^{a b}, T_{t e}^{a a}\right\}, S_{R E}$ is the same as the set defined in section 2.2 because it is assumed that the re-handling operation of a YC is performed without gantry travel.

\subsection{Tchebychev Movement in Perpendicular Block Layout}

The time elements of handling in the operational sequence of a YC during a cycle can be listed as follows: $S_{R E}=\left\{\operatorname{Max}\left(T_{t e}^{a a}, T_{g e}^{a c}\right), t_{h e}^{b c}, t_{p}, t_{h l}^{c b}, \operatorname{Max}\left(T_{t l}^{a a}, T_{g l}^{c a}\right), T_{h l}^{b a}, t_{r}, T_{h e}^{a b}\right\}$. $S_{L}=\left\{\operatorname{Max}\left(T_{t e}^{a a}, T_{g e}^{c a}\right), T_{h e}^{b a}, t_{p}, T_{h l}^{a b}, \operatorname{Max}\left(T_{t l}^{a a}, T_{g l}^{a c}\right), T_{h l}^{b c}, t_{r}, t_{h e}^{c b}\right\}$. $S_{U}=\left\{\operatorname{Max}\left(T_{t e}^{a a}, T_{g e}^{a c}\right), t_{h e}^{b c}, t_{p}, t_{h l}^{c b}, \operatorname{Max}\left(T_{t l}^{a a}, T_{g l}^{c a}\right), T_{h l}^{b a}, t_{r}, T_{h e}^{a b}\right\}$. $S_{D}=\left\{\operatorname{Max}\left(T_{t e}^{a a}, T_{g e}^{c a}\right), \sum_{j=1}^{R_{W H}} T_{U}^{j}, T_{h e}^{b a}, t_{p}, T_{h l}^{a b}, \operatorname{Max}\left(T_{t l}^{a a}, T_{g l}^{a c}\right), t_{h l}^{b c}\right.$, $\left.t_{r}, t_{h e}^{c b}\right\}$, and $S_{R E}^{g e}=\left\{T_{h e}^{b a}, t_{p}, T_{h l}^{a b}, T_{t l}^{a a}, T_{h l}^{b a}, t_{r}, T_{h e}^{a b}, T_{t e}^{a a}\right\}$.

\section{IMPROVING ESTIMATION ACCURA- CIES OF VARIANCE OF CYCLE TIMES OF YARD CRANE}

In the previous sections, we assumed that in an operation, the various time elements are independent of each other. However, precisely speaking, some time elements are dependent on other time elements. In this section, the effect of interdependencies among time ele- 
ments on the variance of the cycle time of various operations is discussed.

For effective description, we use the following notation: $C_{R}(x, y)=$ the cycle time for the receiving operation of a $\mathrm{YC}$, which consists of the handling time elements in $S_{R}$. The block layout, $x$, can be either par (parallel block layout) or per (perpendicular block layout). The movement type, $y$, can be either Rec (rectangular movement) or Tch (Tchebychev movement).

$C_{L}(x, y)=$ the cycle time for the loading operation of a $\mathrm{YC}$, which consists of the handling time elements in $S_{L}$. The block layout and movement type are represented by $x$ and $y$, respectively.

$C_{U}(x, y)=$ the cycle time for the unloading operation of a $\mathrm{YC}$, which consists of the handling time elements in $S_{U}$. The block layout and movement type are represented by $x$ and $y$, respectively.

$C_{D}(x, y)=$ the cycle time for the delivery operation of a $\mathrm{YC}$, which consists of the handling time elements in $S_{D}$. The block layout and movement type are represented by $x$ and $y$, respectively.

\subsection{Considering Interdependencies among Han- dling Elements under Rectangular Movement}

$T_{t e}^{a c}\left(\right.$ or $T_{t e}^{c a}$ ) and $T_{t l}^{c a}$ (or $T_{t l}^{a c}$ ) in sections 2.2 and 2.3 considered the same moving distance of the trolley in the parallel block layout. In addition, $T_{h l}^{b a}$ (or $T_{h l}^{a b}$ ) depends on $T_{h e}^{a b}$ (or $T_{h e}^{b a}$ ). Thus, the variance of the cycle time for the receiving operation, $\operatorname{Var}\left[C_{R}(\operatorname{par}, \operatorname{Rec})\right]$, in a parallel block layout can be modified by including the covariance of those dependent variables and is expressed as follows:

$$
\begin{aligned}
\operatorname{Var}\left[C_{R}\right. & (\operatorname{par}, \operatorname{Rec})]=\operatorname{Var}\left(T_{g e}^{a a}\right) \\
& +\operatorname{Var}\left(T_{t e}^{a c}+T_{t l}^{c a}\right)+\operatorname{Var}\left(T_{h l}^{b a}, T_{h e}^{a b}\right) \\
& =\operatorname{Var}\left(T_{g e}^{a a}\right)+\left(x_{1}+1\right)^{2} \operatorname{Var}\left(T_{t e}^{a c}\right) \\
& +\left(x_{2}+1\right)^{2} \operatorname{Var}\left(T_{h e}^{a b}\right), \\
& \text { where } x_{1}=t_{t l}^{* *} / t_{t e}^{* *} \text { and } x_{2}=t_{h l}^{* *} / t_{h e}^{* *} .
\end{aligned}
$$

In a perpendicular block layout, a YC travels twice between a transfer point and a bay for storing/retrieving a container. This means that $T_{g e}^{a c}$ and $T_{g l}^{c a}$ are interdependent. $T_{h e}^{a b}$ also depends on $T_{h l}^{b a}$ as in the case of a parallel block layout. However, the travel time of a loaded trolley is independent of the travel time of an empty trolley; this is because of our assumption that a truck randomly selects a transfer point. Thus, the cycle time variance for the receiving operation, $\operatorname{Var}\left[C_{R}(\operatorname{per}, \operatorname{Rec})\right]$, in a perpendicular layout can be expressed as follows:

$$
\begin{aligned}
\operatorname{Var}\left[C_{R}\right. & (\text { per }, R e c)]=\operatorname{Var}\left(T_{g e}^{a c}+T_{g l}^{c a}\right)+\operatorname{Var}\left(T_{t e}^{a a}\right) \\
& +\operatorname{Var}\left(T_{t l}^{a a}\right)+\operatorname{Var}\left(T_{h l}^{b a}, T_{h e}^{a b}\right) \\
& =\left(x_{3}+1\right)^{2} \operatorname{Var}\left(T_{g e}^{a c}\right)+\operatorname{Var}\left(T_{t e}^{a a}\right)+\operatorname{Var}\left(T_{t l}^{a a}\right) \\
& +\left(x_{2}+1\right)^{2} \operatorname{Var}\left(T_{h e}^{a b}\right), \\
& \text { where } x_{1}=t_{t l}^{* *} / t_{t e}^{* *} \text { and } x_{2}=t_{h l}^{* *} / t_{h e}^{* *} .
\end{aligned}
$$

Property 1: The variance of the operation cycle time considering the dependencies among handling elements is larger than that without considering the dependencies. The difference in the variance for each type of operation is

$2 x_{1} \operatorname{Var}\left(T_{t e}^{a c}\right)+2 x_{2} \operatorname{Var}\left(T_{h e}^{a b}\right)$ for the parallel layout (receiving, discharging, and loading operations),

$2 x_{1} \operatorname{Var}\left(T_{t e}^{a c}\right)+2 x_{2} \operatorname{Var}\left(T_{h e}^{a b}\right)+E\left(R_{W H}\right)\left[2 x_{1} \operatorname{Var}\left(T_{t e}^{a a}\right)+4 x_{2} \operatorname{Var}\left(T_{h e}^{a b}\right)\right]$ for the parallel layout (delivery operations),

$2 x_{2} \operatorname{Var}\left(T_{h e}^{a b}\right)+2 x_{3} \operatorname{Var}\left(T_{g e}^{a c}\right)$ for the perpendicular layout (receiving, discharging, and loading operations), and $2 x_{2} \operatorname{Var}\left(T_{h e}^{a b}\right)+2 x_{3} \operatorname{Var}\left(T_{g e}^{a c}\right)+E\left(R_{W H}\right)\left[2 x_{1} \operatorname{Var}\left(T_{t e}^{a a}\right)+4 x_{2} \operatorname{Var}\left(T_{h e}^{a b}\right)\right]$ for the perpendicular layout (delivery operations),

where $x_{1}=t_{t l}^{* *} / t_{t e}^{* *}, x_{2}=t_{h l}^{* *} / t_{h e}^{* *}$, and $x_{3}=t_{g l}^{* *} / t_{g e}^{* *}$.

Proof: The proof of this property is simple; however, we omit it in this paper.

\subsection{Considering Interdependencies among Han- dling Elements under Tchebychev Movement}

Section 2.4 shows the time elements of a $\mathrm{YC}$ under Tchebychev movement in a parallel block layout. The random variables $\operatorname{Max}\left(T_{t e}^{a c}, T_{g e}^{a a}\right)$ and $T_{h l}^{b a}$ are dependent on the random variables $T_{t l}^{c a}$ and $T_{h e}^{a b}$, respectively. Thus, the variance of the cycle time for the receiving operation including the covariance, $\operatorname{Var}\left[C_{R}(\mathrm{per}, T c h)\right]$, can be derived as follows.

$$
\begin{aligned}
& \operatorname{Var}\left[C_{R}(\operatorname{par}, T c h)\right]=\operatorname{Var}\left[\operatorname{Max}\left(T_{t e}^{a c}, T_{g e}^{a a}\right)+T_{t l}^{c a}\right] \\
& +\operatorname{Var}\left(T_{h l}^{b a}, T_{h e}^{a b}\right)=\operatorname{Var}\left[\operatorname{Max}\left(T_{t e}^{a c}, T_{g e}^{a a}\right)\right]+x_{1}^{2} \operatorname{Var}\left(T_{t e}^{a c}\right) \\
& +2 \operatorname{Cov}\left[\operatorname{Max}\left(T_{t e}^{a c}, T_{g e}^{a a}\right), T_{t l}^{c a}\right]+\left(x_{2}+1\right)^{2} \operatorname{Var}\left(T_{h e}^{a b}\right), \\
& \text { where } x_{1}=t_{t l}^{* *} / t_{t e}^{* *} \text { and } x_{2}=t_{h l}^{* *} / t_{h e}^{* *} .
\end{aligned}
$$

$E\left[\operatorname{Max}\left(T_{t^{*}}^{a c}, T_{g e}^{a a}\right) T_{t^{*}}^{c a}\right]$ can be derived by using the cumulative distribution functions of $T_{t^{*}}^{c c}$ and $\operatorname{Max}\left(T_{t^{*}}^{a c}, T_{g e}^{a a}\right)$. The detailed expression is derived in Appendix I. In the estimation of the cycle time variance for loading operations, three random variables are mutually dependent on each other:

$\operatorname{Max}\left(T_{t e}^{c a}, T_{g e}^{a a}\right) N\left(1 / l_{0}\right), T_{t l}^{a c}$, and $T_{t e}^{c a}\left(1-N\left(1 / l_{0}\right)\right)$. The random variable $N$ is used to represent the frequency of the loading operation discussed in section 2.2, which is independent of both $\operatorname{Max}\left(T_{t^{*}}^{c a}, T_{g^{*}}^{a a}\right)$ and $T_{t^{*}}^{a c}$. Thus, the variance of the cycle time for the loading operation, $\operatorname{Var}\left[C_{L}(\right.$ par, $T c h)]$ in a parallel block layout can be derived as fol- 
lows. The expressions of the three types of covariance are derived in Appendix II.

$$
\begin{aligned}
& \operatorname{Var}\left[C_{L}(\operatorname{par}, T c h)\right]=\operatorname{Var}\left[\operatorname{Max}\left(T_{t e}^{c a}, T_{g e}^{a a}\right) N\left(1 / l_{0}\right)\right. \\
& \left.+T_{t l}^{a c}+T_{t e}^{c a}\left(1-N\left(1 / l_{0}\right)\right)\right]+\operatorname{Var}\left(T_{h e}^{b a}, T_{h l}^{a b}\right) \\
& =\operatorname{Var}\left[\operatorname{Max}\left(T_{t e}^{c a}, T_{g e}^{a a}\right) N\left(1 / l_{0}\right)+x_{1}^{2} \operatorname{Var}\left(T_{t e}^{c a}\right)\right. \\
& +\operatorname{Var}\left[T_{t e}^{c a}\left(1-N\left(1 / l_{0}\right)\right)\right] \\
& +2 \operatorname{Cov}\left[\operatorname{Max}\left(T_{t e}^{c a}, T_{g e}^{a a}\right) N\left(1 / l_{0}\right), T_{t l}^{a c}\right] \\
& +2 \operatorname{Cov}\left[\operatorname{Max}\left(T_{t e}^{c a}, T_{g e}^{a a}\right) N\left(1 / l_{0}\right), T_{t e}^{c a}\left(1-N\left(1 / l_{0}\right)\right)\right] \\
& +2 \operatorname{Cov}\left[T_{t l}^{a c}, T_{t e}^{c a}\left(1-N\left(1 / l_{0}\right)\right)\right] \\
& +\left(x_{2}+1\right)^{2} \operatorname{Var}\left(T_{h e}^{b a}\right), \\
& \quad \text { where } x_{1}=t_{t l}^{* *} / t_{t e}^{* *} \text { and } x_{2}=t_{h l}^{* *} / t_{h e}^{* *} .
\end{aligned}
$$

In a perpendicular block layout, $\operatorname{Max}\left(T_{t e}^{a a}, T_{g e}^{a c}\right)$ and $\operatorname{Max}\left(T_{t l}^{a a}, T_{g l}^{c a}\right)$ are dependent on each other. Thus, the variance of the cycle time for the receiving operation, $\operatorname{Var}\left[C_{R}(p e r, T c h)\right]$ in a perpendicular block layout can be expressed as follows:

$$
\begin{gathered}
\operatorname{Var}\left[C_{R}(\operatorname{per}, T c h)\right]=\operatorname{Var}\left[\operatorname{Max}\left(T_{t e}^{a a}, T_{g e}^{a c}\right)\right. \\
\left.+\operatorname{Max}\left(T_{t l}^{a a}, T_{g l}^{c a}\right)\right]+\operatorname{Var}\left(T_{h l}^{b a}, T_{h e}^{a b}\right) \\
=\operatorname{Var}\left[\operatorname{Max}\left(T_{t e}^{a a}, T_{g e}^{a c}\right)+\operatorname{Max}\left(T_{t l}^{a a}, T_{g l}^{a c}\right)\right] \\
+2 \operatorname{Cov}\left[\operatorname{Max}\left(T_{t e}^{a a}, T_{g e}^{a c}\right), \operatorname{Max}\left(T_{t l}^{a a}, T_{g l}^{c a}\right)\right] \\
+\left(x_{2}+1\right)^{2} \operatorname{Var}\left(T_{t e}^{a b}\right), \\
\text { where } x_{2}=t_{h l}^{* *} / t_{h e}^{* *} .
\end{gathered}
$$

Property 2: Under the Tchebychev movement in both parallel and perpendicular block layouts, for every type of operation, the variance of the operation cycle time considering the dependencies among handling elements is larger than that without considering the dependencies. It is larger by a value equal to the sum of the covariance of trolley and gantry movements and $2 x_{2} \operatorname{Var}\left(T_{h e}^{a b}\right)$, where $x_{2}=t_{h l}^{* *} / t_{h e}^{* *}$.

Proof: (1) For receiving operations, the difference is the sum of $2 \operatorname{Cov}\left[\operatorname{Max}\left(T_{t e}^{a c}, T_{g e}^{a a}\right), T_{t l}^{c a}\right]$ and $2 x_{2} \operatorname{Var}\left(T_{h e}^{a b}\right)$ in the case of the parallel block layout. For a perpendicular block layout, it is the sum of $2 \operatorname{Cov}\left[\operatorname{Max}\left(T_{t e}^{a a}, T_{g e}^{a c}\right), \operatorname{Max}\left(T_{t l}^{a a}, T_{g l}^{c a}\right)\right]$ and $2 x_{2} \operatorname{Var}\left(T_{h e}^{a b}\right)$.

(2) For loading operations, the difference is the sum of $2 \operatorname{Cov}\left[\operatorname{Max}\left(T_{t e}^{c a}, T_{g e}^{a a}\right) N\left(1 / l_{0}\right), T_{t l}^{c a}\right], 2 \operatorname{Cov}\left[\operatorname{Max}\left(T_{t e}^{c a}, T_{g e}^{a a}\right) N\left(1 / l_{0}\right)\right.$, $\left.T_{t e}^{c a}\left(1-N\left(1 / l_{0}\right)\right)\right], 2 \operatorname{Cov}\left[T_{t l}^{c a}, T_{t e}^{c a}\left(1-N\left(1 / l_{0}\right)\right)\right]$ and $2 x_{2} \operatorname{Var}\left(T_{h e}^{b a}\right)$ in the parallel block layout. In the perpendicular block layout, it is the sum of $2 \operatorname{Cov}\left[\operatorname{Max}\left(T_{t e}^{a a}, T_{g e}^{c a}\right), \operatorname{Max}\left(T_{t l}^{a a}, T_{g l}^{a c}\right)\right]$ and $2 x_{2} \operatorname{Var}\left(T_{h e}^{b a}\right)$.

(3) For unloading operations, it is the sum of $2 \operatorname{Cov}[\operatorname{Max}$ $\left.\left(T_{t e}^{c a}, T_{g e}^{a a}\right) N\left(1 / u_{W H}\right), T_{t l}^{a c}\right], 2 \operatorname{Cov}\left[\operatorname{Max}\left(T_{t e}^{a c}, T_{g e}^{a a}\right) N\left(1 / u_{W H}\right), T_{t e}^{a c}\right.$
$\left.\left(1-N\left(1 / u_{W H}\right)\right)\right]$, and $2 x_{2} \operatorname{Var}\left(T_{h e}^{a b}\right)$ in the parallel block layout. In the perpendicular block layout, it is the sum of $2 \operatorname{Cov}\left[\operatorname{Max}\left(T_{t e}^{a a}, T_{g e}^{a c}\right), \operatorname{Max}\left(T_{t l}^{a a}, T_{g l}^{c a}\right)\right]$ and $2 x_{2} \operatorname{Var}\left(T_{h e}^{a b}\right)$.

(4) For delivery operations, it is the sum of $2 \operatorname{Cov}[\operatorname{Max}$ $\left.\left(T_{t e}^{c a}, T_{t l}^{a c}\right)\right], 2 x_{2} \operatorname{Var}\left(T_{h e}^{b a}\right)$, and $E\left(R_{W H}\right)\left[2 x_{1} \operatorname{Var}\left(T_{t e}^{a a}\right)+4 x_{2} \operatorname{Var}\right.$ $\left.\left(T_{h e}^{a b}\right)\right]$ in the parallel block layout. In the perpendicular block layout, it is the sum of $2 \operatorname{Cov}\left[\operatorname{Max}\left(T_{t e}^{a a}, T_{g e}^{c a}\right), \operatorname{Max}\right.$ $\left.\left(T_{t l}^{a a}, T_{g l}^{a c}\right)\right], 2 x_{2} \operatorname{Var}\left(T_{h e}^{b a}\right)$, and $E\left(R_{W H}\right)\left[2 x_{1} \operatorname{Var}\left(T_{t e}^{a a}\right)+4 x_{2} \operatorname{Var}\right.$ $\left.\left(T_{h e}^{a b}\right)\right] . E\left(R_{W H}\right)\left[2 x_{1} \operatorname{Var}\left(T_{t e}^{a a}\right)+4 x_{2} \operatorname{Var}\left(T_{h e}^{a b}\right)\right]$ is for the re-handling time. Here, $x_{1}=t_{t l}^{* *} / t_{t e}^{* *}$.

\section{NUMERICAL EXPERIMENTS}

A numerical experiment is conducted for comparing the effect of interdependencies among the handling elements for the two types of block layouts and the two traveling modes. It is assumed that $t_{g e}^{b b}=t_{g l}^{b b}=1.18 \mathrm{~min}$, $t_{g e}^{b c}=t_{g l}^{b c}=1.22 \mathrm{~min}, t_{t e}^{b b}=t_{t l}^{b b}=0.15 \mathrm{~min}, t_{t e}^{b c}=t_{t l}^{b c}=0.19 \mathrm{~min}$, $t_{h e}^{b b}=0.25 \mathrm{~min}, t_{h l}^{b b}=0.42 \mathrm{~min}, t_{h e}^{b c}=0.20 \mathrm{~min}, t_{h l}^{b c}=0.34$ min, $t_{p}=t_{r}=5 \mathrm{~s}$, and $l_{0}=10$. A block is assumed to have 34 bays, 6 tiers, and 9 rows.

Table 1 and Table 2 show the proportion of covariance in the variance value. In the parallel block layout, the covariance occupies a larger proportion for the loading and unloading operations than for the receiving and delivery operations. This may result from a higher proportion of trolley movement in the cycle time of loading or unloading operations. In the perpendicular block layout, the covariance accounts for about half of the entire variance for all the types of operations except the delivery operation. This might be because gantry travel time constitutes a high proportion of the cycle time, which has a high covariance between empty and loaded travel for the receiving, loading, and discharging operations. The relocation operation occupies a high proportion of the cycle time for delivery operations. For all types of operations, the covariance is larger for rectangular movement than that for Tchebychev movement. Note that the proportion of covariance corresponds to the error of the estimator for the variance without considering the interdependence among the handling elements in a cycle.

The expected queue length is one of the important performance measures for container terminals, and it is affected by the cycle time variances for various operations. By using the expected values and variances of the cycle times derived in this paper, it is possible to estimate the expected queue lengths of trucks at the yard for various truck arrival rates. The average waiting time of a truck at a block is given by the well-known PollaczekKhintchine (P-K) formula for an M/G/1 queuing system (Gross and Harris, 1998):

$$
W_{q}=\frac{\rho E(T)}{2(1-\rho)}\left(1+\frac{\operatorname{Var}(T)}{E^{2}(T)}\right)
$$


Table 1. Proportion of Covariance in Cycle Time Variance for Rectangular Movement.

\begin{tabular}{|c|l|l|l|l|l|l|l|c|}
\hline & \multicolumn{2}{|c|}{ Receiving operations } & \multicolumn{2}{c|}{ Loading operations } & \multicolumn{2}{c|}{ Unloading operations } & \multicolumn{2}{c|}{ Delivery operations } \\
\cline { 2 - 9 } & Parallel & Perpendicular & Parallel & Perpendicular & Parallel & Perpendicular & Parallel & Perpendicular \\
\hline \hline Variance $_{1}{ }^{*}$ & $0.092 \mathrm{~min}$ & $0.247 \mathrm{~min}$ & $0.036 \mathrm{~min}$ & $0.247 \mathrm{~min}$ & $0.019 \mathrm{~min}$ & $0.247 \mathrm{~min}$ & $2.048 \mathrm{~min}$ & $2.203 \mathrm{~min}$ \\
\hline Variance $_{2}{ }^{* *}$ & 0.106 & 0.490 & 0.050 & 0.490 & 0.032 & 0.490 & 2.090 & 2.474 \\
\hline Covariance & 0.013 & 0.243 & 0.013 & 0.243 & 0.013 & 0.243 & 0.041 & 0.271 \\
\hline $\begin{array}{l}\text { Covariance } \\
\text { ratio*** }\end{array}(\%)$ & 12.549 & 49.614 & 26.749 & 49.614 & 40.846 & 49.614 & 1.972 & 10.956 \\
\hline
\end{tabular}

Note) ${ }^{*}$ Variance $_{1}$ is the value under the assumption that the time elements are independent of each other; ${ }^{* *}$ Variance $_{2}$ is the value wherein mutual independences among the time elements are considered; ${ }^{* * *}$ The covariance ratio is the ratio of covariance to variance $_{2}$.

Table 2. Proportion of the Covariance in Cycle Time Variance for Tchebychev Movement.

\begin{tabular}{|c|l|l|l|l|l|l|l|l|}
\hline & \multicolumn{2}{|c|}{ Receiving operations } & \multicolumn{2}{c|}{ Loading operations } & \multicolumn{2}{c|}{ Unloading operations } & \multicolumn{2}{c|}{ Delivery operations } \\
\cline { 2 - 9 } & Parallel & Perpendicular & Parallel & Perpendicular & Parallel & Perpendicular & Parallel & Perpendicular \\
\hline \hline Variance $_{1}$ & $0.073 \mathrm{~min}$ & $0.211 \mathrm{~min}$ & $0.022 \mathrm{~min}$ & $0.211 \mathrm{~min}$ & $0.016 \mathrm{~min}$ & $0.211 \mathrm{~min}$ & $2.029 \mathrm{~min}$ & $2.167 \mathrm{~min}$ \\
\hline Variance $_{2}$ & 0.084 & 0.420 & 0.026 & 0.420 & 0.027 & 0.420 & 2.068 & 2.404 \\
\hline Covariance & 0.011 & 0.209 & 0.005 & 0.209 & 0.011 & 0.209 & 0.039 & 0.237 \\
\hline $\begin{array}{c}\text { Covariance } \\
\text { ratio(\%) }\end{array}$ & 12.764 & 49.853 & 17.285 & 49.853 & 41.410 & 49.853 & 1.871 & 9.877 \\
\hline
\end{tabular}

Table 3. Comparison of Expected Truck Waiting Times for Four Types of Operations between Two Types of Variances in Parallel Block Layout.

\begin{tabular}{|l|l|l|l|l|l|l|}
\hline \multicolumn{2}{|c|}{$\begin{array}{c}\text { Truck arrival rate } \\
\text { Operation type }\end{array}$} & \multicolumn{1}{|c|}{0.05} & \multicolumn{1}{c|}{0.1} & \multicolumn{1}{c|}{0.15} & \multicolumn{1}{c|}{0.2} & \multicolumn{1}{l|}{0.25} \\
\hline \hline \multirow{2}{*}{ Receiving } & Without covariance & $0.065 \mathrm{~min}$ & $0.141 \mathrm{~min}$ & $0.233 \mathrm{~min}$ & $0.345 \mathrm{~min}$ & $0.484 \mathrm{~min}$ \\
operations & With covariance & 0.065 & 0.142 & 0.234 & 0.346 & 0.486 \\
& Difference & $0.448 \%$ & $0.448 \%$ & $0.448 \%$ & $0.448 \%$ & $0.448 \%$ \\
\hline \multirow{2}{*}{ Loading } & Without covariance & $0.043 \mathrm{~min}$ & $0.093 \mathrm{~min}$ & $0.150 \mathrm{~min}$ & $0.217 \mathrm{~min}$ & $0.296 \mathrm{~min}$ \\
operations & With covariance & 0.043 & 0.093 & 0.150 & 0.217 & 0.297 \\
& Difference & $0.283 \%$ & $0.283 \%$ & $0.283 \%$ & $0.283 \%$ & $0.283 \%$ \\
\hline \multirow{2}{*}{ Unloading } & Without covariance & $0.041 \mathrm{~min}$ & $0.089 \mathrm{~min}$ & $0.143 \mathrm{~min}$ & $0.207 \mathrm{~min}$ & $0.282 \mathrm{~min}$ \\
operations & With covariance & 0.042 & 0.089 & 0.144 & 0.208 & 0.284 \\
& Difference & $0.728 \%$ & $0.728 \%$ & $0.728 \%$ & $0.728 \%$ & $0.728 \%$ \\
\hline \multirow{2}{*}{ Delivery } & Without covariance & $0.279 \mathrm{~min}$ & $0.665 \mathrm{~min}$ & $1.232 \mathrm{~min}$ & $2.148 \mathrm{~min}$ & $3.877 \mathrm{~min}$ \\
operations & With covariance & 0.280 & 0.668 & 1.237 & 2.157 & 3.892 \\
& Difference & $0.402 \%$ & $0.402 \%$ & $0.402 \%$ & $0.402 \%$ & $0.402 \%$ \\
\hline
\end{tabular}

Table 4. Comparison of Expected Truck Waiting Times for Four Types of Operations between Two Types of Variances in Perpendicular Block Layout.

\begin{tabular}{|l|l|l|l|l|l|l|}
\hline \multicolumn{2}{|c|}{$\begin{array}{c}\text { Truck arrival rate } \\
\text { Operation type }\end{array}$} & \multicolumn{1}{c|}{0.05} & \multicolumn{1}{c|}{0.1} & \multicolumn{1}{c|}{0.15} & \multicolumn{1}{c|}{0.2} & \multicolumn{1}{c|}{0.25} \\
\hline \hline \multirow{2}{*}{ Receiving } & Without covariance & $0.145 \mathrm{~min}$ & $0.332 \mathrm{~min}$ & $0.580 \mathrm{~min}$ & $0.929 \mathrm{~min}$ & $1.452 \mathrm{~min}$ \\
operations & With covariance & 0.151 & 0.345 & 0.604 & 0.967 & 1.511 \\
& Difference & $4.062 \%$ & $4.062 \%$ & $4.062 \%$ & $4.062 \%$ & $4.062 \%$ \\
\hline \multirow{2}{*}{ Loading } & Without covariance & $0.145 \mathrm{~min}$ & $0.332 \mathrm{~min}$ & $0.580 \mathrm{~min}$ & $0.929 \mathrm{~min}$ & $1.452 \mathrm{~min}$ \\
operations & With covariance & 0.151 & 0.345 & 0.604 & 0.967 & 1.511 \\
& Difference & $4.062 \%$ & $4.062 \%$ & $4.062 \%$ & $4.062 \%$ & $4.062 \%$ \\
\hline \multirow{2}{*}{ Unloading } & Without covariance & $0.145 \mathrm{~min}$ & $0.332 \mathrm{~min}$ & $0.580 \mathrm{~min}$ & $0.929 \mathrm{~min}$ & $1.452 \mathrm{~min}$ \\
operations & With covariance & 0.151 & 0.345 & 0.604 & 0.967 & 1.511 \\
& Difference & $4.062 \%$ & $4.062 \%$ & $4.062 \%$ & $4.062 \%$ & $4.062 \%$ \\
\hline \multirow{2}{*}{ Delivery } & Without covariance & $0.427 \mathrm{~min}$ & $1.079 \mathrm{~min}$ & $2.201 \mathrm{~min}$ & $4.579 \mathrm{~min}$ & $13.022 \mathrm{~min}$ \\
operations & With covariance & 0.434 & 1.097 & 2.238 & 4.656 & 13.241 \\
& Difference & $1.681 \%$ & $1.681 \%$ & $1.681 \%$ & $1.681 \%$ & $1.681 \%$ \\
\hline
\end{tabular}


rate of trucks multiplied by the expected cycle time of a YC). Note that $E(T)$ is the expected cycle time of an operation, and $\operatorname{Var}(T)$ is its variance. Of course, the expected waiting times vary across different types of operations.

Table 3 and Table 4 show a comparison of the expected waiting times of trucks for the four types of operations associated with various arrival rates of trucks $(0.05,0.1,0.15,0.2$, and 0.25$)$. It was assumed that the YC travels under the Tchebychev metric. As expected, trucks experience longer average waiting times than other cases when the interdependencies among the handling elements are considered for the evaluation of the cycle time variances. However, the average difference between the two cases over all types of operation and all truck arrival rates is only $0.47 \%$ and $3.47 \%$ for the parallel and perpendicular block layouts, respectively.

\section{CONCLUSIONS}

The cycle time of a $\mathrm{YC}$ includes the gantry travel time between yard bays, vertical movement (hoisting/ lowering) time of the spreader, and trolley movement time. A cycle of each of the four different types of operations was divided into detailed elements of handling. For each of these elements, expressions were derived for the expectations and variances of the handling cycle times. The interdependencies among the handling elements of a $\mathrm{YC}$ were considered for deriving the exact expressions for the variances of operation cycle times. Two types of block layouts (parallel and perpendicular), two types of movement models (rectangular and Tchebychev), and four types of operations (receiving, delivery, unloading, and loading operations) were analyzed.

From the analysis of the expressions for the variance of cycle times and the results of numerical experiments, it was shown that the effect of the interdependencies among the handling elements was larger in the cases of receiving, loading, and discharging operations than for delivery operations. Further, it was found that the effect of the interdependencies among the handling elements was larger in the case of the perpendicular layout than in case of the parallel layout. In the numerical experiment, it was found that the differences between the values of variances considering the interdependencies among the handling elements and those without considering them reached up to almost $50 \%$. However, when two different values of variances were used to estimate the expected waiting time for trucks, which is one of the most important performance measures, the difference was almost negligible (an average of $0.47 \%$ and $3.47 \%$ for the two types of layouts). This result shows that the variances estimated by considering the independencies among handling elements are valid, and they can be applied to future studies related to cycle times.

\section{ACKNOWLEDGMENTS}

This study was supported by the Korean Ministry of Education and Human Resources Development through the Regional Research Centers Program (Research Center for Logistics Information Technology).

\section{REFERENCES}

Bozer, Y. A. and White, J. A. (1984), Travel time models for automated storage/retrieval systems, IIE Transactions, 16(4), 329-338.

Bozer, Y. A. and White, J. A. (1990), Design and performance models for end-of-aisle order picking systems, Management Science, 36(7), 852-866.

Chen, T. (1999), Yard operations in the container terminal-a study in the 'unproductive moves', Maritime Policies and Management, 26(1), 27-38.

de Castilho, B. and Daganzo, C. F. (1993), Handling strategies for import containers at marine terminals, Transportation Research, 27B(2), 151-166.

Gross, D. and Harris, C. M. (1998), Fundamentals of Queueing Theory 3rd ed., Wiley-Interscience, New York.

Hu, Y. H., Huang, S. Y., Chen, C., Hsu, W. J., Toh, A. C., Loh, C. K., and Song, T. (2005), Travel time analysis of a new automated storage and retrieval system, Computers and Operations Research, 32 (6), 1515-1544.

Hwang, H. and Lee, S. B. (1990), Travel-time models considering the operating characteristics of the storage/retrieval system, International Journal of Production Research, 28(10), 1779-1789.

Kim, K. H. (1997), Evaluation of the number of rehandles in container yards, Computers and Industrial Engineering, 32 (4), 701-711.

Kim, K. Y. (2006), Evaluation models for the container handling times of the automated transfer crane in container terminal, IE Interfaces, 19(3), 214-224.

Le-Duc, T. (2005), Design and control of efficient order picking processes, Ph.D. Dissertation, Erasmus University Rotterdam, Netherlands.

Lee, B. K. and Kim, K. H. (2010a), Comparison and Evaluation of Various Cycle-Time Models for Yard Cranes in Container Terminals, International Journal of Production Economics, 126(2), 350-360.

Lee, B. K. and Kim, K. H. (2010b), Optimizing the block size in container yards, Transportation Research, 46E(1), 120-135.

Vidovic, M. and Kim, K. H. (2006), Estimating the cycle time of three-stage material handling systems, Annals of Operations Research, 144(1), 181-200. 
APPENDIX I: $E\left[\operatorname{Max}\left(T_{t^{*}}^{a c}, T_{g^{*}}^{a a d}\right)\right] T_{t^{*}}^{c a}$ for deriving the expression of $\operatorname{Var}\left[C_{R}(\right.$ par,$\left.T C h)\right]$

$T_{t^{*}}^{c a}$ is the movement time of the trolley between two random positions, each of which follows $U(0, q)$, where $q$ is a shape factor and can be expressed as $t_{t^{*}}^{c b} / t_{g^{*}}^{b b} \cdot T_{g^{*}}^{a a}$. is the travel time of a $\mathrm{YC}$ between two random positions, each of which follows $U(0,1)$. Note that $T_{t^{*}}^{c a}$ and $T_{g^{*}}^{a a}$ are independent with each other. The probability density functions of the two random variables are $f_{t^{*}}^{c a}(x)=1 / q(0 \leq x$ $\leq q), 1(q<x \leq 1)$ and $f_{g^{*}}^{a a}(y)=2-2 y$, respectively.

$$
\begin{aligned}
& E\left[\operatorname{Max}\left(T_{t^{*}}^{a c}, T_{g^{*}}^{a a}\right) T_{t^{*}}^{c a}\right] \\
& =\int_{y} \int_{x}[\operatorname{Max}(x, y) x] f r_{t^{*}}^{c a} r_{g^{*}}^{a a}(x, y) d x d y \\
& =\int_{0}^{q}\left[\int_{0}^{y} x y f f_{t^{*}}^{c a}(x) d x+\int_{y}^{q} x^{2} f r_{t^{*}}^{c a}(x) d x\right] f r_{g^{*}}^{c a}(y) d y \\
& =\int_{q}^{1}\left[\int_{0}^{q} x y f f_{t^{*}}^{c a}(x) d x\right] f r_{g^{*}}^{a a}(y) d y \\
& =\frac{1}{6} q+\frac{1}{4} q^{3}-\frac{1}{15} q^{4} .
\end{aligned}
$$

APPENDIX II: $\operatorname{Cov}\left[\operatorname{Max}\left(T_{t^{*}}^{c a}, T_{g^{*}}^{a a}\right) N\left(1 / l_{0}\right), T_{t^{*}}^{a c}\right], \operatorname{Cov}$ $\left[\operatorname{Max}\left(T_{t^{*}}^{c a}, T_{g^{*}}^{a a}\right) N\left(1 / l_{0}\right), T_{t^{*}}^{c a}\left(1-N\left(1 / l_{0}\right)\right)\right]$, and $\operatorname{Cov}\left[T_{t^{*}}^{a c}, T_{t^{*}}^{c a}\right.$ $\left.\left(1-N\left(1 / l_{0}\right)\right)\right]$ for deriving the expression of $\operatorname{Var}\left[C_{L}\right.$ $\operatorname{Var}\left[C_{L}(\right.$ par,$\left.T c h)\right]$

1) Expression for $\operatorname{Cov}\left[\operatorname{Max}\left(T_{t^{*}}^{c a}, T_{g^{*}}^{a a}\right) N\left(1 / l_{0}\right), T_{t^{*}}^{a c}\right]$

$$
\begin{aligned}
& \operatorname{Cov}\left[\operatorname{Max}\left(T_{t^{*}}^{c a}, T_{g^{*}}^{a a}\right) N\left(1 / l_{0}\right), T_{t^{*}}^{a c}\right] \\
& =E\left[\operatorname{Max}\left(T_{t^{*}}^{c a}, T_{g^{*}}^{a a}\right) N\left(1 / l_{0}\right), T_{t^{*}}^{a c}\right] \\
& -E\left[\operatorname{Max}\left(T_{t^{*}}^{c a}, T_{g^{*}}^{a a}\right) N\left(1 / l_{0}\right)\right] E\left[T_{t^{*}}^{a c}\right]
\end{aligned}
$$

However, the random variable $N\left(1 / l_{0}\right)$ is independent of both $T_{t^{*}}^{c a}$ and $T_{g^{*}}^{a a}$. Therefore,

$E\left[\operatorname{Max}\left(T_{t^{*}}^{c a}, T_{g^{*}}^{a a}\right) N\left(1 / l_{0}\right) T_{t^{*}}^{a c}\right]$ is the same as

$E\left[\operatorname{Max}\left(T_{t^{*}}^{c a}, T_{g^{*}}^{a a}\right) T_{t^{*}}^{a c}\right] E\left[N\left(1 / l_{0}\right)\right]$, and
$E\left[\operatorname{Max}\left(T_{t^{*}}^{c a}, T_{g^{*}}^{a a}\right) N\left(1 / l_{0}\right)\right] E\left[T_{t^{*}}^{a c}\right]$ is the same as $E\left[\operatorname{Max}\left(T_{t^{*}}^{c a}, T_{g^{*}}^{a a}\right)\right] E\left[N\left(1 / l_{0}\right)\right] E\left[T_{t^{*}}^{a c}\right]$.

$E\left[\operatorname{Max}\left(T_{t^{*}}^{c a}, T_{g^{*}}^{a a}\right) T_{t^{*}}^{a c}\right]$ has already been derived in Appendix I.

2) Expression for $\operatorname{Cov}\left[\operatorname{Max}\left(T_{t^{*}}^{c a}, T_{g^{*}}^{a a}\right) N\left(1 / l_{0}\right), T_{t^{*}}^{a c}(1-N\right.$ $\left.\left.N\left(1 / l_{0}\right)\right)\right]$

$$
\begin{aligned}
& \operatorname{Cov}\left[\operatorname{Max}\left(T_{t^{*}}^{c a}, T_{g^{*}}^{a a}\right) N\left(1 / l_{0}\right), T_{t^{*}}^{c a}\left(1-N\left(1 / l_{0}\right)\right)\right] \\
& =E\left[\operatorname{Max}\left(T_{t^{*}}^{c a}, T_{g^{*}}^{a a}\right) N\left(1 / l_{0}\right) T_{t^{*}}^{c a}\left(1-N\left(1 / l_{0}\right)\right)\right] \\
& =E\left[\operatorname{Max}\left(T_{t^{*}}^{c a}, T_{g^{*}}^{a a}\right) N\left(1 / l_{0}\right)\right] E\left[T_{t^{*}}^{c a}\left(1-N\left(1 / l_{0}\right)\right)\right]
\end{aligned}
$$

$T_{t^{*}}^{c a}, T_{t^{*}}^{a a}$, and $N\left(1 / l_{0}\right)$ are independent of each other. Thus, $E\left[\operatorname{Max}\left(T_{t^{*}}^{c a}, T_{g^{*}}^{a a}\right) N\left(1 / l_{0}\right) T_{t^{*}}^{c a}\left(1-N\left(1 / l_{0}\right)\right)\right]$ and $E[\operatorname{Max}$ $\left(T_{t^{*}}^{c a}, T_{g^{*}}^{a a}\right) N\left(1 / l_{0}\right) E\left[T_{t^{*}}^{c a}\left(1-N\left(1 / l_{0}\right)\right)\right]$ can be substituted with $E\left[\operatorname{Max}\left(T_{t^{*}}^{c a}, T_{g^{*}}^{a a}\right) T_{t^{*}}^{c a}\right] E\left(N\left(1 / l_{0}\right)\left(1-N\left(1 / l_{0}\right)\right)\right]$ and $E\left[\operatorname{Max}\left(T_{t^{*}}^{c a}\right.\right.$, $\left.\left.\left.T_{g^{*}}^{a a}\right)\right] E\left[N\left(1 / l_{0}\right)\right] E\left[T_{t^{*}}^{c a}\right] E\left(1-N\left(1 / l_{0}\right)\right)\right]$.

We have already defined $E\left[N\left(1 / l_{0}\right)\right.$ and $\operatorname{Var}\left[N\left(1 / l_{0}\right)\right]$ in section 2.2 as $E\left[N\left(1 / l_{0}\right)\right]=1 / l_{0}$ and $\operatorname{Var}\left[N\left(1 / l_{0}\right)\right]=\left(l_{0}-1\right) /$ $l_{0}^{2}$, respectively. Thus, $E\left[N\left(1 / l_{0}\right)\left(1-N\left(1 / l_{0}\right)\right)\right]=E\left[N\left(1 / l_{0}\right)\right]$ $-E\left[N\left(1 / l_{0}\right)^{2}\right]=E\left[N\left(1 / l_{0}\right)\right]\left(1-E\left[N\left(1 / l_{0}\right)\right]-\operatorname{Var}\left[N\left(1 / l_{0}\right)\right]=0\right.$.

3) Expression for $\operatorname{Cov}\left[T_{t^{*}}^{c a}, T_{t^{*}}^{a a}\left(1-N\left(1 / l_{0}\right)\right)\right]$

$$
\begin{aligned}
& \operatorname{Cov}\left[T_{t^{*}}^{a c}, T_{t^{*}}^{c a}\left(1-N\left(1 / l_{0}\right)\right)\right] \\
& =E\left[T_{t^{*}}^{a c} T_{t^{*}}^{c a}\left(1-N\left(1 / l_{0}\right)\right)\right] \\
& -E\left[T_{t^{*}}^{a c}\right] E\left[T_{t^{*}}^{c a}\left(1-N\left(1 / l_{0}\right)\right)\right]
\end{aligned}
$$

$T_{t^{*}}^{a c}$ and $N\left(1 / l_{0}\right)$ are independent of each other. Thus, $E$ $\left[\left(T_{t^{*}}^{a c}\right)^{2}\left(1-N\left(1 / l_{0}\right)\right)\right]=E\left[\left(T_{t^{*}}^{a c}\right)^{2}\right] E\left[1-N\left(1 / l_{0}\right)\right]$ and $E\left[T_{t^{*}}^{a c}\right] E$ $\left[T_{t^{*}}^{c a}\left(1-N\left(1 / l_{0}\right)\right)\right]=E^{2}\left[T_{t^{*}}^{a c}\right] E\left[1-N\left(1 / l_{0}\right)\right]$. Therefore, Equation (II.3) can be summarized as $\operatorname{Var}\left[T_{t^{*}}^{a c}\right] E\left[1-N\left(1 / l_{0}\right)\right]$. 